\title{
Role of Immunotherapy in Triple-Negative Breast Cancer
}

Tanya E. Keenan, MD, MPH, ${ }^{1,2}$ and Sara M. Tolaney, MD, MPH ${ }^{1,2}$

\section{ABSTRACT}

Immune checkpoint inhibitors (ICls) have led to durable clinical remissions in many metastatic cancers. However, the single-agent efficacy of $\mathrm{ICl}$ in breast cancer is low, including in triple-negative breast cancer (TNBC), which has several key characteristics that enhance ICI responses. Strategies to improve anticancer immune responses in TNBC are urgently needed to extend survival for patients with metastatic disease. This review presents $\mathrm{ICl}$ monotherapy response rates and discusses combination strategies with chemotherapy, targeted therapies, and novel immunotherapies. It concludes with a summary of immunotherapy biomarkers in TNBC and a call to action for future directions of research critical to advancing the efficacy of immunotherapy for patients with TNBC.

J Natl Compr Canc Netw 2020;18(4):479-489 doi: 10.6004/jncen.2020.7554

${ }^{1}$ Department of Medical Oncology, Dana-Farber Cancer Institute, and

${ }^{2}$ Harvard Medical School, Boston, Massachusetts.
Triple-negative breast cancer (TNBC), defined by the lack of estrogen and progesterone receptors and HER2, accounts for $15 \%$ to $20 \%$ of all breast cancers and typically displays aggressive behavior, including earlier recurrence and metastasis. ${ }^{1}$ The predominant systemic therapy for most metastatic TNBC (mTNBC) is chemotherapy, but responses are often short-lived, and patients have a median overall survival (OS) of 12 to 18 months. ${ }^{1}$ Therefore, improved therapies are urgently needed.

Immunotherapy has prolonged survival in other solid tumors and represents a promising treatment strategy for TNBC. The most successful immunotherapeutic agents consist of immune checkpoint inhibitors (ICIs), which block immunosuppressive receptors, such as cytotoxic T lymphocyte antigen-4 (CTLA-4) and PD-1, to improve the cytotoxicity and proliferative capacity of tumorinfiltrating lymphocytes (TILs). ICIs, including monoclonal antibodies against PD-1 (ie, pembrolizumab, nivolumab), PD-L1 (ie, atezolizumab, durvalumab, avelumab), and CTLA-4 (ie, ipilimumab), have generated durable responses across many tumor types. ${ }^{2-9}$

Several key characteristics make TNBC more likely to respond to immunotherapy than other breast cancer subtypes. First, TNBC has more TILs, ${ }^{10}$ which correlate with better responses to ICIs in other tumors, ${ }^{11}$ and high levels of TILs in TNBC associate with improved prognosis in early-stage TNBC. ${ }^{12}$ Second, TNBC has higher levels of PD-L1 expression on both tumor and immune cells, ${ }^{13,14}$ providing direct targets for ICIs and also correlating with response to anti-PD-1 therapies in other tumors. ${ }^{2}$ Finally, TNBC has a greater number of nonsynonymous mutations, ${ }^{15}$ which give rise to tumor-specific neoantigens that activate neoantigen-specific $\mathrm{T}$ cells to mount an antitumor immune response, ${ }^{16}$ which can be strengthened by ICIs. ${ }^{17}$

This review presents a framework for understanding the current clinical experience surrounding ICIs and other novel immunotherapy agents in TNBC (Figure 1). In addition, emerging immunotherapy biomarkers are discussed and future directions for immunotherapy development in TNBC are outlined.

\section{ICI Monotherapy}

Although response rates to ICIs are higher in TNBC than in hormone receptor-positive and HER2-positive breast cancers, the single-agent efficacy is still low, with monotherapy response rates ranging from approximately $5 \%$ in 


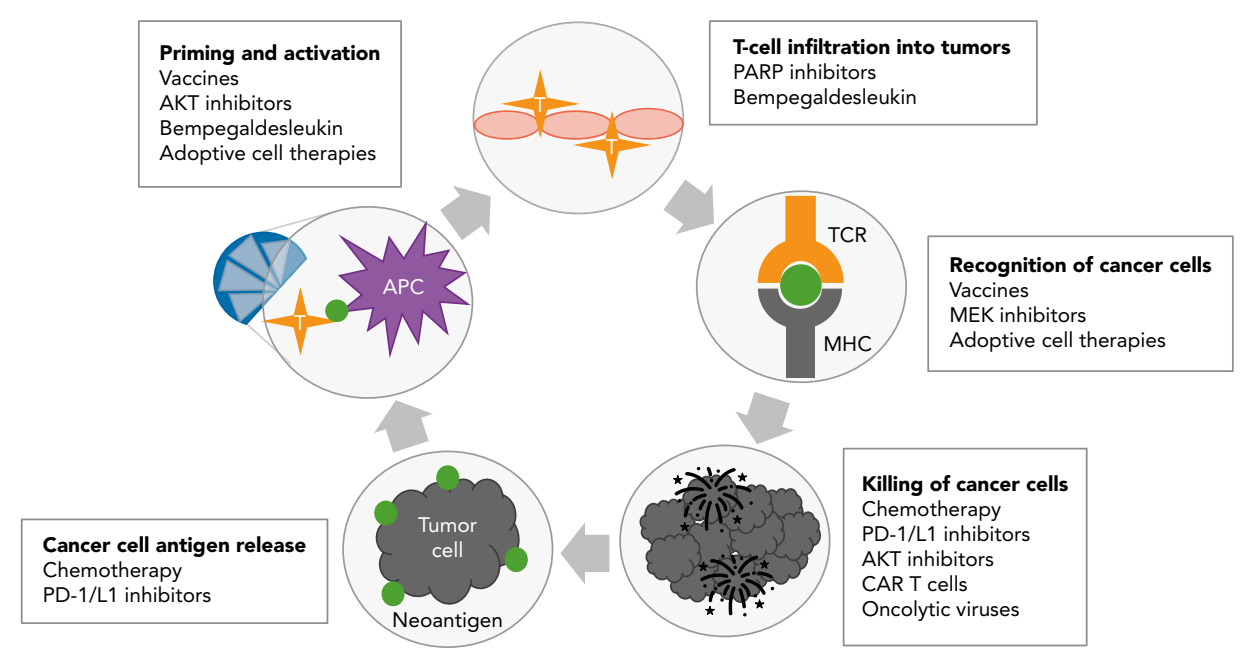

Figure 1. Immunotherapy and combination agents in triple-negative breast cancer.

unselected patients to approximately $23 \%$ in treatmentnaïve PD-L1-positive patients (Table 1). The initial phase Ib KEYNOTE-012 study (ClinicalTrials.gov identifier: NCT01848834) of the PD-1 inhibitor pembrolizumab demonstrated an encouraging overall response rate (ORR) of $18.5 \%$ in 32 patients with both pretreated and treatmentnaïve PD-L1-positive mTNBC. ${ }^{18}$ However, the subsequent larger phase II KEYNOTE-086 study (NCT02447003) found an ORR of $5.3 \%$ in 170 patients with PD-L1-unselected pretreated tumors. ${ }^{19}$ Notably, in the 84 treatment-naïve patients on this trial, the ORR was $21.4 \%$, suggesting that ICIs have greater efficacy in the first-line metastatic setting. ${ }^{20}$ In support of this concept, the phase III KEYNOTE-119 trial (NCT02555657) in patients with pretreated mTNBC did not show an improvement in ORR, progression-free survival (PFS), or OS with single-agent pembrolizumab versus single-agent chemotherapy, although patients with the highest levels of tumor PD-L1 expression showed a trend toward greater benefit with pembrolizumab. ${ }^{21}$

The PD-L1 inhibitors avelumab and atezolizumab have also been explored as ICI monotherapy in mTNBC. Avelumab demonstrated an ORR of $5.2 \%$ in 58 heavily pretreated patients in the phase Ib JAVELIN trial (NCT01772004), ${ }^{22}$ and the phase I trial of atezolizumab (NCT01375842) led to an ORR of $10 \%$ in 115 pretreated patients, with no responses seen in the PD-L1-negative subgroup. ${ }^{23}$ Both trials underscore the limited singleagent efficacy of ICIs in mTNBC, with particularly low response rates observed in pretreated metastatic disease.

\section{Metastatic Chemotherapy \\ Combination Regimens}

Combination regimens of PD-1/L1 inhibitors plus chemotherapy have demonstrated more success in mTNBC than single-agent ICIs (Table 1). Among early trials including both PD-L1-positive and PD-L1-negative tumors, pembrolizumab with eribulin, a microtubule inhibitor that suppresses transforming growth factor $\beta$ (TGF- $\beta$ ), ${ }^{24}$ which has been associated with intratumoral CD8-positive T-cell exclusion, ${ }^{25}$ yielded an ORR of $26.4 \%$ in 106 patients treated with 0 to 2 prior lines of therapy for metastatic disease in the ENHANCE-1 trial (ClinicalTrials.gov identifier: NCT02513472). ${ }^{26}$ Similarly, the smaller phase Ib trial of atezolizumab and nab-paclitaxel (NCT01375842) showed a promising ORR of $39.4 \%$ in 33 patients, also treated with 0 to 2 prior lines, ${ }^{27}$ perhaps related to the ability of taxanes to promote toll-like receptor activity and dendritic cell activation..$^{28}$ The subsequent randomized IMpassion130 phase III trial (NCT02425891) in treatmentnaïve mTNBC showed that atezolizumab added to nab-paclitaxel resulted in a clinically meaningful OS improvement of 7 months in the PD-L1-positive subgroup but not in the entire cohort, ${ }^{29}$ which is notable because the trial's hierarchical statistical design required overall benefit to formally test the subgroup effect. ${ }^{28}$ These results led the FDA and the European Commission to approve atezolizumab and nab-paclitaxel in PD-L1-positive mTNBC, establishing the first immunotherapy approval in breast cancer.

Several ongoing trials will further elucidate the role of chemotherapy in combination with ICIs in mTNBC (Table 2). The KEYNOTE-355 trial (NCT02819518) recently announced in a press release that first-line chemotherapy with pembrolizumab significantly improved PFS compared with chemotherapy alone in patients with mTNBC expressing PD-L1 using the 22C3 antibody with a combined positive score $\geq 10$, defined as the ratio of all PD-L1-expressing cells, including tumor cells, lymphocytes, and macrophages, to the number of all tumor cells. ${ }^{30}$ In addition, 2 trials of chemotherapy with or without atezolizumab are currently accruing. The IMpassion131 


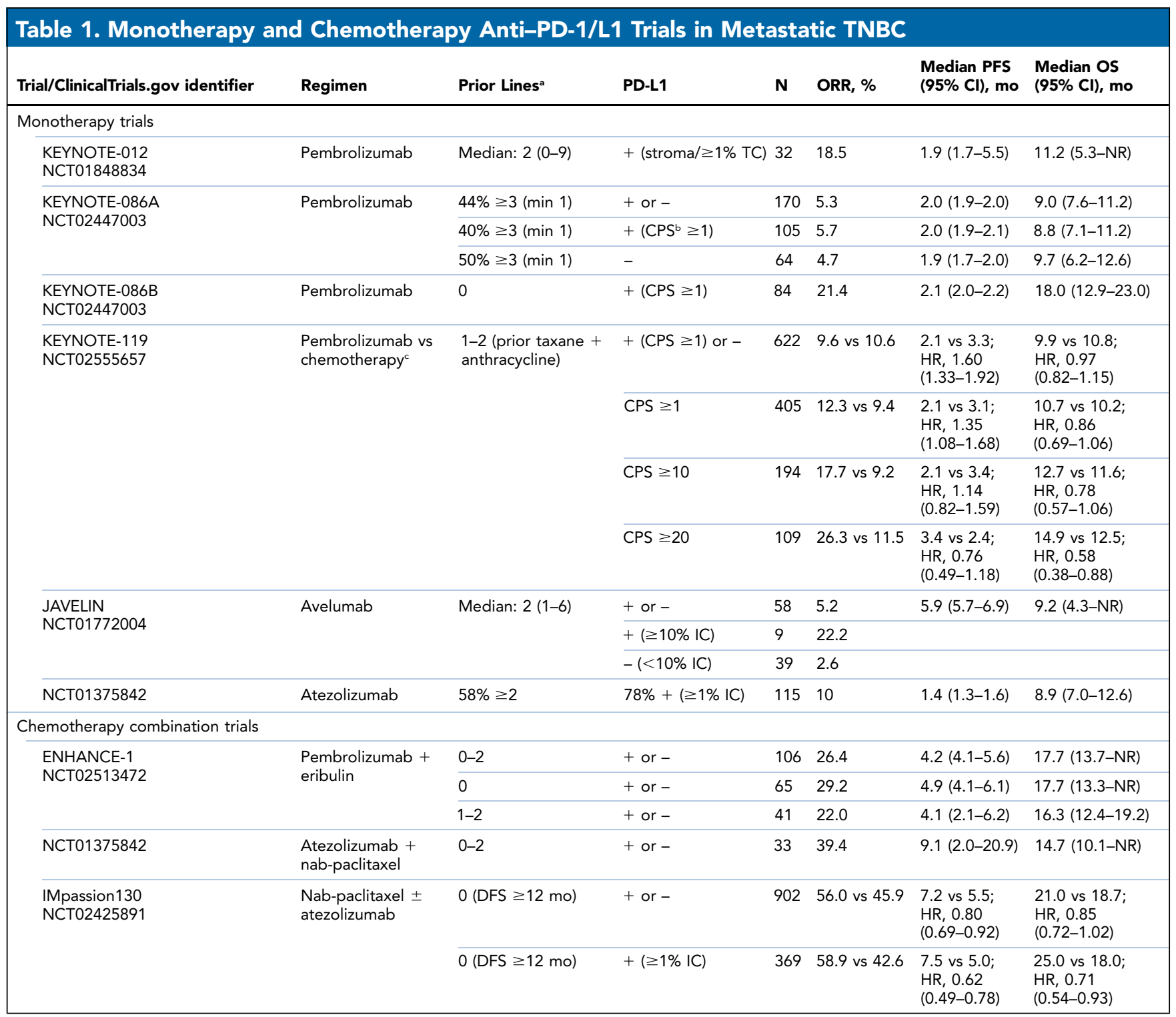

Abbreviations: CPS, combined positive score; DFS, disease-free survival; HR, hazard ratio: IC, immune cells; IHC, immunohistochemistry; min, minimum; NR, not reached; ORR, overall response rate; OS, overall survival; PFS, progression-free survival; TC, tumor cells; TNBC, triple-negative breast cancer.

aprior lines of systemic therapy for metastatic disease.

bPD-L1 IHC 22C3 = (number of PD-L1-positive cells measured by the immunohistochemistry 22C3 assay [tumor cells, lymphocytes, macrophages]/total number of viable tumor cells) $\times 100$.

'Capecitabine, eribulin, gemcitabine, or vinorelbine.

trial (NCT03125902) will investigate whether first-line atezolizumab combined with paclitaxel will improve outcomes compared with paclitaxel alone, whereas IMpassion132 (NCT03371017) will determine whether first-line atezolizumab combined with chemotherapy will improve outcomes compared with chemotherapy alone in TNBC recurring within 1 year of adjuvant therapy.

\section{Early-Stage Chemotherapy Combination Regimens}

The combination of ICIs with chemotherapy in earlystage TNBC has also demonstrated preliminary success
(Table 3). Among patients with stage II-III disease, the I-SPY 2 trial (ClinicalTrials.gov identifier: NCT01042379) initially revealed that pembrolizumab added to neoadjuvant paclitaxel followed by doxorubicin and cyclophosphamide nearly tripled the estimated pathologic complete response (pCR) rates from $22 \%$ to $60 \%,{ }^{31}$ perhaps related to the known immunostimulatory effects of anthracyclines to increase intratumoral immune effectors, ${ }^{32}$ as well as antigen uptake and processing. ${ }^{33}$ However, the GeparNuevo trial (NCT02685059), which included earlier-stage Tlb and higher disease, demonstrated that the addition of durvalumab to neoadjuvant 


\section{Table 2. Ongoing Anti-PD-1/L1 Combination and Novel Immunotherapy Trials in TNBC}

\begin{tabular}{|c|c|c|c|c|}
\hline $\begin{array}{l}\text { Trial/ } \\
\text { ClinicalTrials.gov Identifier }\end{array}$ & Regimen & Lines ${ }^{\mathrm{a}}$ or Stage & $\begin{array}{l}\text { Primary } \\
\text { Endpoint }\end{array}$ & $\mathbf{N}$ \\
\hline \multicolumn{5}{|c|}{ Chemotherapy combination trials } \\
\hline $\begin{array}{l}\text { KEYNOTE-355 } \\
\text { NCT02819518 }\end{array}$ & $\begin{array}{l}\text { Chemotherapy (carboplatin }+ \text { gemcitabine, or } \\
\text { nab-/paclitaxel) } \pm \text { pembrolizumab }\end{array}$ & 0 (DFS $\geq 6 \mathrm{mo}$ ) & PFS, OS & 847 \\
\hline $\begin{array}{l}\text { IMpassion131 } \\
\text { NCT03125902 }\end{array}$ & Paclitaxel \pm atezolizumab & 0 & PFS & 600 \\
\hline $\begin{array}{l}\text { IMpassion } 132 \\
\text { NCT03371017 }\end{array}$ & $\begin{array}{l}\text { Chemotherapy (carboplatin }+ \text { gemcitabine, or } \\
\text { capecitabine) } \pm \text { atezolizumab }\end{array}$ & 0 (DFS < 12 mo) & OS & 350 \\
\hline \multicolumn{5}{|c|}{ Targeted therapy combination trials } \\
\hline $\begin{array}{l}\text { ETCTN } \\
\text { NCT02849496 }\end{array}$ & $\begin{array}{l}\text { Olaparib (PARPi) } \pm \text { atezolizumab: } \\
\text { BRCAm-positive }\end{array}$ & any & PFS & 72 \\
\hline $\begin{array}{l}\text { DORA } \\
\text { NCT03167619 }\end{array}$ & $\begin{array}{l}\text { Olaparib (PARPi) } \pm \text { durvalumab: sporadic or } \\
\text { germline } B R C A m\end{array}$ & $\leq 2$ including current platinum & PFS & 60 \\
\hline $\begin{array}{l}\text { IPATunity } 130 \\
\text { NCT03337724 }\end{array}$ & Paclitaxel \pm ipatasertib $(\mathrm{AKTi}) \pm$ atezolizumab & 0 (DFS $\geq 12 \mathrm{mo})$ & PFS & 450 \\
\hline $\begin{array}{l}\text { BEGONIA } \\
\text { NCT03742102 }\end{array}$ & $\begin{array}{l}\text { Paclitaxel }+ \text { durvalumab } \pm \text { capivasertib (AKTi) or } \\
\text { danvatirsen (STAT3i) or oleclumab (anti-CD73) }\end{array}$ & 0 & $\mathrm{AE}$ rate & 120 \\
\hline $\begin{array}{l}\text { InCITe } \\
\text { NCT03971409 }\end{array}$ & $\begin{array}{l}\text { Avelumab + binimetinib (MEKi) or utomilumab } \\
\text { (IgG2 antibody) or anti-OX40 antibody }\end{array}$ & $0-3$ & ORR & 150 \\
\hline \multicolumn{5}{|c|}{ Novel immunotherapy agent trials } \\
\hline $\begin{array}{l}\text { I-SPY } 2 \\
\text { NCT01042379 }\end{array}$ & $\begin{array}{l}\text { Paclitaxel } \pm \text { pembrolizumab } \times 4+\text { SD }-101 \times \\
6 \rightarrow \mathrm{AC} \times 4 \rightarrow \text { surgery }\end{array}$ & Stage II-III & Estimated pCR & TBD \\
\hline NCT03362060 & $\begin{array}{l}\text { PVX-410 vaccine }+ \text { pembrolizumab: HLA- } \\
\text { A2-positive }\end{array}$ & $>1$ & Immune ${ }^{b}$ & 20 \\
\hline NCT02826434 & Adjuvant PVX-410 vaccine + durvalumab & Stage II-III & $\mathrm{AE}$ rate & 22 \\
\hline NCT03012100 & Cyclophosphamide \pm folate receptor $\alpha$ vaccine & $\geq \mathrm{T} 1 \mathrm{c} / \geq \mathrm{N} 1 / \mathrm{RCB}$ & DFS & 280 \\
\hline NCT03199040 & $\begin{array}{l}\text { Adjuvant neoantigen DNA vaccine } \pm \\
\text { durvalumab }\end{array}$ & RCB post NACT & Safety & 24 \\
\hline NCT03606967 & $\begin{array}{l}\text { Gemcitabine }+ \text { carboplatin } \times 18 \text { weeks } \rightarrow \text { nab- } \\
\text { paclitaxel }+ \text { durvalumab } \pm \text { neoantigen vaccine }\end{array}$ & 0 & PFS & 70 \\
\hline NCT03004183 & $\begin{array}{l}\text { Adenovirus-mediated expression of HSV } \\
\text { thymidine kinase }+ \text { valacyclovir }+ \text { SBRT } \rightarrow \\
\text { pembrolizumab }\end{array}$ & $>1$ & ORR & 57 \\
\hline NCT04111510 & Autologous TILs (LN-145) with intravenous IL-2 & $0-3$ & ORR, AEs & 10 \\
\hline NCT02792114 & $\begin{array}{l}\text { Cyclophosphamide } \rightarrow \text { mesothelin-targeted } \\
\text { CAR T cells }\end{array}$ & $>1$ & MTD & 36 \\
\hline
\end{tabular}

Abbreviations: AC, doxorubicin/cyclophosphamide; $A E$, adverse event; BRCAm, BRCA mutation; DFS; disease-free survival; HSV, herpes simplex virus; MTD, maximum tolerated dose; NACT, neoadjuvant chemotherapy; ORR, overall response rate; OS, overall survival; PARPi, PARP inhibitor; PCR, pathologic complete response; PFS, progression-free survival; RCB, residual cancer burden; SBRT, stereotactic body radiation therapy; TBD, to be determined; TILs, tumor infiltration lymphocytes; TNBC, TNBC, triple-negative breast cancer.

aprior lines of systemic therapy for metastatic disease.

bFold activation of peripheral $\mathrm{T}$ cells.

nab-paclitaxel followed by epirubicin and cyclophosphamide did not significantly improve pCR rates in the overall trial and only improved pCR rates when durvalumab was started 2 weeks before chemotherapy, which was a subgroup analysis underpowered for significance testing. $^{34}$ The KEYNOTE-522 trial (NCT03036488) reported that pembrolizumab added to paclitaxel plus carboplatin and then to an anthracycline plus cyclophosphamide as neoadjuvant therapy, followed by surgery plus an additional 9 cycles of adjuvant pembrolizumab, improved pCR rates from $51.2 \%$ to $64.8 \%$, and 18 -month event-free survival (EFS) rates from $85.3 \%$ to $91.3 \%$ (hazard ratio [HR], $0.63 ; 95 \% \mathrm{CI}, 0.43-0.93$ ). ${ }^{35}$ These results need to be confirmed with extended follow-up data and weighed against long-term immune-related toxicity. In this trial, less benefit was seen in node-negative patients, underscoring the conclusion that the risks of this 5 -agent regimen may outweigh the benefits for patients with early-stage node-negative TNBC and TNBC with high TIL levels, who have excellent outcomes with current standardof-care regimens. ${ }^{12}$

Most recently, the NeoTRIPaPDL1 trial (NCT02620280) of atezolizumab added to neoadjuvant carboplatin plus 


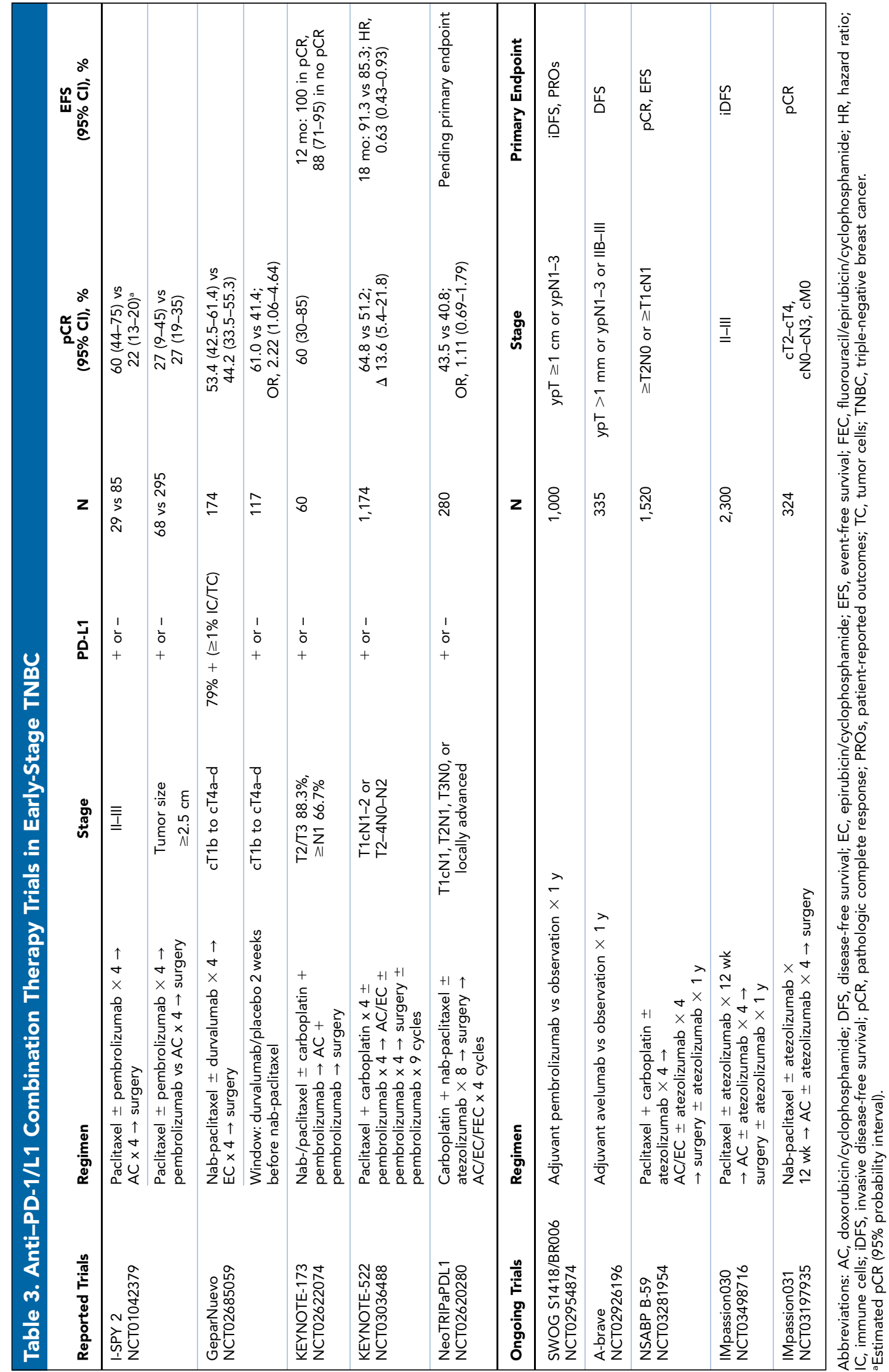


nab-paclitaxel followed by adjuvant cyclophosphamide plus an anthracycline without atezolizumab reported that pCR was not significantly different in patients with early high-risk or locally advanced TNBC treated with versus without atezolizumab (43.5\% vs $40.8 \%$; $P=.66$ ) (Table 3).$^{36}$ Similarly, the 8-cycle pembrolizumab cohort without doxorubicin and cyclophosphamide in the neoadjuvant I-SPY 2 trial demonstrated that neoadjuvant pembrolizumab for 4 cycles added to paclitaxel followed by neoadjuvant pembrolizumab alone for 4 additional cycles had an equivalent estimated pCR rate of $27 \%$ compared with neoadjuvant paclitaxel, doxorubicin, and cyclophosphamide in patients with high-risk early-stage TNBC. ${ }^{37}$ Although this regimen did not meet the target threshold pCR rate of $60 \%$ required for graduation per I-SPY 2 criteria, these results suggest there may be potential strategies for de-escalation from the 5-agent KEYNOTE-522 regimen that should be further explored. Overall, the contrasting results of KEYNOTE-522 may be explained by different chemotherapy backbones, including the presence of an anthracycline based on the results of the TONIC trial, which showed the superiority of doxorubicin as an induction agent to enhance sensitivity to PD-1 blockade. ${ }^{38}$ The dissimilar results could also be due to differences in ICI activity, given that PD-1 inhibitors but not PD-L1 inhibitors block PD-L2 inhibitory signaling, but it is unclear whether this mechanistic difference translates into differences in clinical efficacy. ${ }^{39}$

Several ongoing early-stage disease trials will further clarify the efficacy of ICIs in neoadjuvant and adjuvant regimens for TNBC (Table 3). Two key trials are investigating whether 1 year of adjuvant anti-PD-1/L1 therapy prolongs EFS or disease-free survival (DFS): the SWOG S1418/BR006 trial (NCT02954874) of pembrolizumab for patients with residual disease, and the A-brave trial (NCT02926196) of avelumab for patients with high-risk or residual disease. Similarly, 2 large trials are addressing whether the addition of atezolizumab to both neoadjuvant and adjuvant therapy prolongs EFS or invasive DFS: the placebo-controlled NSABP B-59 trial (NCT03281954) of atezolizumab added to neoadjuvant 4 -agent chemotherapy followed by 1 year of adjuvant atezolizumab, and the IMpassion030 trial (NCT03498716) of atezolizumab added to neoadjuvant 3-agent chemotherapy followed by 1 year of adjuvant atezolizumab or observation. Finally, one additional smaller trial is exploring whether atezolizumab added to neoadjuvant chemotherapy alone improves outcomes. The IMpassion031 trial (NCT03197935) will assess whether atezolizumab versus placebo added to neoadjuvant nab-paclitaxel, doxorubicin, and cyclophosphamide results in higher pCR rates.

\section{Targeted Therapy Combination Regimens}

Certain targeted therapy combinations are emerging as potential strategies to overcome innate resistance to PD-1/L1 inhibitors in mTNBC (Table 4). Preclinical evidence in BRCA-deficient models of TNBC suggests that PARP inhibitors increase cytoplasmic DNA and activate the STING (stimulator of interferon genes) protein, which increases type 1 interferons and T-cell intratumoral infiltration. ${ }^{40}$ Translational studies of human breast cancers have also shown that BRCA1/2 mutations negatively associate with markers of immunogenicity, such as immune infiltration and T-cell-mediated cytolysis, the latter calculated as the summed expression of the CD8-positive T-cell effectors $P R F 1$ and GZMA. ${ }^{41}$ To evaluate the clinical efficacy of combining the PARP inhibitor niraparib with the PD-1 inhibitor pembrolizumab, the phase II TOPACIO trial (ClinicalTrials.gov identifier: NCT02657889) enrolled 55 patients with advanced or mTNBC and found an ORR of $47 \%$ and a median PFS of 8.3 months among the 15 patients with tumor BRCA mutations. ${ }^{42}$ Within the limits of cross-trial comparisons, this ORR was slightly lower than the ORRs of $55 \%$ and $62 \%$ associated with single-agent PARP inhibitor therapy in patients with TNBC and germline BRCA mutations in the OlympiAD (NCT02000622) $^{43}$ and EMBRACA ${ }^{44}$ (NCT01945775) trials, respectively. However, the median PFS was longer than those observed in the TNBC subgroups of these trials, which were 5.5 and 5.8 months, respectively. The MEDIOLA trial (NCT02734004) of olaparib and durvalumab in mTNBC with germline BRCA mutations demonstrated an ORR of $58.8 \%$, more similar to single-agent PARP therapy, with a median PFS of 4.9 months. ${ }^{45}$ The nonrandomized designs, different PARP inhibitors, and small sample sizes of these initial trials limit definitive conclusions. Ongoing larger trials of olaparib with atezolizumab (ETCTN trial; NCT02849496) or durvalumab (DORA trial; NCT03167619) are in progress (Table 2), as well as an I-SPY 2 cohort of neoadjuvant olaparib with durvalumab \pm paclitaxel followed by neoadjuvant doxorubicin and cyclophosphamide (NCT01042379).

AKT inhibitors constitute another important class of targeted therapy agents currently in development with ICIs in TNBC. Loss of the tumor suppressor PTEN, a negative regulator of AKT, has been linked to immunotherapy resistance, ${ }^{46,47}$ and AKT inhibition augments expansion of tumor-specific lymphocytes with a memory cell phenotype. ${ }^{48}$ Preliminary data from a phase Ib trial of taxane chemotherapy plus the AKT inhibitor ipatasertib and atezolizumab demonstrated an impressive ORR of $73 \%$ in the first 26 patients, with similar responses irrespective of PIK3CA/AKT1/PTEN alteration status and PD-L1 expression (Table 4). ${ }^{49}$ 


\begin{tabular}{|c|c|c|c|c|c|c|c|}
\hline $\begin{array}{l}\text { Trial/ } \\
\text { ClinicalTrials.gov Identifier }\end{array}$ & Regimen & Prior Lines $^{\mathrm{a}}$ & Biomarker & $\mathbf{N}$ & ORR, \% & $\begin{array}{l}\text { Median PFS } \\
(95 \% \mathrm{Cl}) \text {, mo }\end{array}$ & $\begin{array}{l}\text { Median OS } \\
(95 \% \mathrm{Cl}) \text {, mo }\end{array}$ \\
\hline \multicolumn{8}{|c|}{ Targeted therapy combination trials } \\
\hline \multicolumn{8}{|l|}{ PARP inhibitors } \\
\hline \multirow{2}{*}{$\begin{array}{l}\text { TOPACIO/ } \\
\text { KEYNOTE-162 } \\
\text { NCT02657889 }\end{array}$} & & & $B R C A m+$ & 15 & 47 & $8.3(2.1-\mathrm{NR})$ & \\
\hline & & & $B R C A m-$ & 27 & 11 & $2.1(1.4-2.5)$ & \\
\hline $\begin{array}{l}\text { MEDIOLA } \\
\text { NCT02734004 }\end{array}$ & $\begin{array}{l}\text { Olaparib }+ \\
\text { durvalumab after } \\
4 \text { wk run-in }\end{array}$ & $\leq 2$ & Germline BRCAm & 17 & 58.8 & 4.9 & 20.5 \\
\hline \multicolumn{8}{|l|}{ MEK inhibitors } \\
\hline $\begin{array}{l}\text { COLET } \\
\text { NCT02322814 }\end{array}$ & $\begin{array}{l}\text { Nab-paclitaxel vs } \\
\text { paclitaxel }+ \\
\text { cobimetinib }+ \\
\text { atezolizumab }\end{array}$ & 0 & PD-L1 + or - & 90 & $\begin{array}{l}29.0 \text { vs } \\
34.4\end{array}$ & $\begin{array}{l}7.0(3.7-12.8) \text { vs } \\
3.8(3.0-7.4)\end{array}$ & $\begin{array}{l}\text { NR (10.2-NR) vs } \\
11.0(9.5-\mathrm{NR})\end{array}$ \\
\hline \multicolumn{8}{|c|}{ Novel immunotherapy agent trials } \\
\hline \multicolumn{8}{|l|}{ IL-2 agonists } \\
\hline $\begin{array}{l}\text { PIVOT-02 } \\
\text { NCT02983045 }\end{array}$ & $\begin{array}{l}\text { NKTR-214 + } \\
\text { nivolumab }\end{array}$ & $0-2$ & PD-L1 + or - & 38 & 13.2 & & \\
\hline \multicolumn{8}{|l|}{ CAR T cells } \\
\hline NCT01837602 & $\begin{array}{l}\text { Intratumoral c-MET } \\
\text { mRNA CAR T cells }\end{array}$ & Any & PD-L1 + or - & 6 & 0 & & \\
\hline
\end{tabular}

Abbreviations: BRCAm, BRCA mutation; DFS, disease-free survival; NR, not reached; ORR, overall response rate; OS, overall survival; PFS, progression-free survival; TNBC, triple-negative breast cancer.

aPrior lines of systemic therapy for metastatic disease.

These data led to the addition of a paclitaxel, ipatasertib, and atezolizumab arm in the larger phase III IPATunity130 trial (NCT03337724), and the AKT inhibitor capivasertib is being explored in combination with paclitaxel and durvalumab in the BEGONIA trial (NCT03742102; Table 2).

Considerable interest also surrounds MEK inhibitors in combination with checkpoint blockade. Preclinical studies in TNBC showed that MEK inhibition upregulates PD-L1 and major histocompatibility complex expression and enhances the antitumor immune response to PD-1/L1 inhibition..$^{50}$ The phase II COLET trial (NCT02322814), which investigated the MEK inhibitor cobimetinib, the PD-L1 inhibitor atezolizumab, and taxane chemotherapy in 63 patients with treatment-naïve mTNBC, showed that this triplet combination therapy had modest activity with ORRs of $34.4 \%$ and $29.0 \%$ and median PFS of 3.8 and 7.0 months in the paclitaxel and nab-paclitaxel cohorts, respectively (Table 4). ${ }^{51}$ However, this trial was not designed to determine the incremental efficacy of adding PD-1 blockade to combined MEK inhibition and taxane chemotherapy. ${ }^{51}$ Ongoing (NCT03971409) and future trials of MEK inhibitors combined with PD-1/L1 inhibitors will clarify whether MEK inhibitors enhance responses to checkpoint blockade in TNBC (Table 2).

\section{Novel Immunotherapy Agents}

Novel therapeutic strategies to overcome deficient anticancer immunity in TNBC are urgently needed, and some are already in clinical development. One such strategy is the IL-2 pathway agonist bempegaldesleukin (NKTR-214), which preferentially activates the IL-2b receptor to expand effector $\mathrm{T}$ cells more than regulatory $\mathrm{T}$ cells. ${ }^{52-54}$ The phase I/II PIVOT-02 trial (ClinicalTrials.gov identifier: NCT02983045) evaluated 38 patients with mTNBC treated with bempegaldesleukin in combination with nivolumab and found an ORR of $13.2 \%$, with durable responses regardless of PD-L1 expression (Table 4). Future trials of bempegaldesleukin combined with anti-PD-1/L1 therapies and chemotherapy in TNBC are currently being developed. Another immune priming strategy is the 
intratumoral toll-like receptor 9 (TLR9) agonist SD-101, which stimulates plasmacytoid dendritic cells to release interferon- $\alpha$ and mature into antigen-presenting cells, thereby increasing CD8+ T-cell infiltration in tumors. ${ }^{55}$ The I-SPY 2 trial (NCT01042379) is currently investigating the estimated pCR impact of neoadjuvant SD-101 and pembrolizumab added to weekly paclitaxel followed by doxorubicin and cyclophosphamide compared with paclitaxel, doxorubicin, and cyclophosphamide alone (Table 2).

Breast cancer vaccines constitute another novel therapeutic strategy to enhance anticancer immunity (Table 2). By presenting breast cancer peptides to $\mathrm{T}$ cells, these vaccines enhance the priming and activation of $\mathrm{T}$ cells and strengthen immune recognition of cancer cells. Multiple trials of vaccines with and without PD-1/L1 inhibitors are currently enrolling patients with TNBC in the adjuvant or metastatic setting, including trials of the PVX-410 vaccine (NCT03362060, NCT02826434), folate receptor $\alpha$ vaccine (NCT03012100), and neoantigen vaccines. The PVX-410 vaccine targets the XBP1 and CD138 peptides that are overexpressed in TNBC. ${ }^{56}$ Similarly, the folate receptor $\alpha$ vaccine also targets a peptide overexpressed in breast cancer, and led to immune responses that persisted at least 12 months in the initial phase I trial of patients with breast and ovarian cancer. ${ }^{57}$

Rather than targeting peptides overexpressed in tumors but also shared by normal cells, neoantigen vaccines target peptides arising from tumor-specific mutations unique to each patient's tumor and not present in normal cells, and therefore T-cell responses to these neoantigens are not limited by self-tolerance. ${ }^{58}$ The randomized phase I trial of a neoantigen vaccine with or without durvalumab is enrolling 24 patients with residual TNBC after neoadjuvant therapy (NCT03199040), ${ }^{59}$ whereas the randomized phase II trial of nab-paclitaxel and durvalumab with or without a neoantigen vaccine is enrolling 70 patients with treatment-naïve $\mathrm{mTNBC}$ who are treated first with gemcitabine and carboplatin for 18 weeks prior to randomization (NCT03606967). ${ }^{60}$ Using patient-specific neoantigens identified and prioritized by next-generation sequencing and epitope prediction algorithms, these vaccines aim to prime $\mathrm{T}$ cells to recognize neoantigens specific to cancer cells. ${ }^{60}$ Whether these breast cancer vaccines will elicit a sufficient antitumor immune response to improve clinical outcomes remains to be determined.

Other novel immunotherapy strategies currently being explored in TNBC include oncolytic viruses and adoptive cell therapies, such as TIL transfer and CAR T cells. Oncolytic viruses are being developed to target and kill TNBC, such as the phase II trial of in situ oncolytic virus therapy consisting of adenovirusmediated expression of herpes simplex virus thymidine kinase plus valacyclovir with stereotactic body radiation therapy followed by pembrolizumab (NCT03004183). Likewise, initial studies of TIL transfer in breast cancer are emerging. These therapies activate and expand TILs removed from a patient's tumor, including $\mathrm{T}$ cells that recognize tumor-specific neoantigens. One patient with chemorefractory hormone receptor-positive metastatic breast cancer experienced durable complete remission after adoptive transfer of TILs reactive against 4 tumorspecific mutant proteins, ${ }^{61}$ and a phase II trial of the autologous TIL transfer therapy LN-145 will enroll 10 patients with mTNBC (NCT04111510).

Finally, CAR T cells, engineered to target tumor antigens, are also being developed in breast cancer, with careful safety measures to prevent potentially lifethreatening adverse events. Although preliminary, a phase 0 trial of mRNA-transfected c-MET CAR T cells delivered intratumorally in 6 patients with metastatic breast cancer found no CAR $\mathrm{T}$ cell adverse effects greater than grade 1 (NCT01837602), ${ }^{62}$ and a phase I trial of mesothelin-targeted CAR $\mathrm{T}$ cells, which demonstrated responses without toxicity in other tumors, ${ }^{63}$ will enroll 36 patients with pretreated metastatic breast cancer (NCT02792114). These novel immunotherapies, although preliminary and requiring extensive clinical validation, will enhance understanding of anticancer immunity in breast cancer and contribute to the development of future effective immunotherapies.

\section{Biomarkers of Immunotherapy Response}

Biomarkers that predict clinical benefit to immunotherapy in TNBC are needed to select patients most likely to benefit from single-agent ICIs, and to develop combination therapies that overcome ICI resistance. Only 2 validated biomarkers currently exist: mismatch repair deficiency and PD-L1 expression on immune cells. ${ }^{64,65}$ However, mismatch repair deficiency occurs rarely in breast cancer and more commonly in early-stage disease, ${ }^{64}$ and most patients with mTNBC are PD-L1negative by the currently approved SP-142 assay. ${ }^{66}$ Furthermore, the utility of PD-L1 expression as a reliable biomarker is limited by several issues: its varying expression over time and metastatic sites; the discrepancy among different PD-L1 assays, particularly when staining immune cells ${ }^{67}$; the observations that some PD-L1-negative patients respond to ICIs ${ }^{68}$; and recent trials in the early disease setting that show little to no correlation of PD-L1 expression with benefit specific to ICIs, including the KEYNOTE-522 and NeoTRIPaPDL1 trials. ${ }^{36,69}$

Emerging potential biomarkers of immunotherapy response in TNBC include high tumor mutational burden (TMB), TILs, and transcriptional signatures of immune infiltration. High TMB with variably defined 
cutoffs correlates with response to PD-1/L1 inhibitors across various cancer types, ${ }^{70-75}$ but the prevalence of high $\mathrm{TMB}>10$ mutations/ $\mathrm{Mb}$ in breast cancer is low: $3 \%$ of primary tumors and $8 \%$ of metastatic tumors. ${ }^{76,77}$ The TAPUR study (NCT02693535) of single-agent pembrolizumab in patients with heavily pretreated metastatic breast cancer with $\mathrm{TMB} \geq 9$ mutations/Mb reported an ORR of $21 \%,^{78}$ which is consistent with improved clinical efficacy of single-agent ICIs in breast cancer with high TMB. In a retrospective study of 62 women with $\mathrm{mTNBC}$ treated with anti-PD-1/L1 therapies, $\mathrm{TMB} \geq 10$ mutations/Mb was also found to be associated with longer PFS following ICI treatment, whereas $P T E N$ alterations were found to be associated with shorter $\mathrm{PFS}^{79}$ These findings require validation in future prospective studies, and the predictive versus prognostic role of PTEN alterations needs to be clarified.

Higher levels of TILs have also been associated with better responses to ICI monotherapy, ${ }^{80}$ and recently TILs $\geq 5 \%$ have been shown to independently predict improved response and survival following pembrolizumab but not chemotherapy in the KEYNOTE-119 trial of pembrolizumab compared with chemotherapy monotherapy in patients with mTNBC treated with 1 to 2 lines of prior systemic therapy, although these results are preliminary and used a one-sided $P$ value for pembrolizumab. ${ }^{81,82}$ Similarly, a study of neoadjuvant durvalumab concurrent with nab-paclitaxel and dosedense doxorubicin and cyclophosphamide in 57 patients with early-stage TNBC found that high CD8 cell density and immune gene expression signatures, as well as DNA damage response signatures, correlated with higher pCR rates, ${ }^{83}$ although these changes were not shown to be specific to ICI therapy compared with chemotherapy alone. Finally, the TONIC trial (NCT02499367) of various induction regimens prior to single-agent nivolumab showed that tumors treated with doxorubicin or cisplatin demonstrated increased expression of genes related to the PD-L1 pathway, T-cell cytotoxicity, and inflammation, suggesting that certain chemotherapy agents induce favorable tumor immune microenvironment changes that promote response to PD-1 blockade. ${ }^{38}$ Additional translational studies are needed in breast cancer to more thoroughly investigate immunotherapy response correlates found in other tumors, such as antigen presentation and interferon- $\lambda$ signaling. ${ }^{73,84}$

\section{Future Directions}

Despite the recent approval of atezolizumab and nabpaclitaxel in PD-L1-positive mTNBC, many unanswered questions remain regarding immunotherapy in TNBC. Critical areas in need of development include immunotherapies for PD-L1-negative mTNBC and TNBC refractory to prior PD-1/L1 therapies, as well as strategies that minimize immune-related toxicity. Altogether, clinical trials based on strong preclinical evidence combined with rich translational studies to understand mechanisms of response and resistance are necessary to advance the clinical development of immunotherapy in TNBC.

\section{Acknowledgments}

We kindly thank Kate Bifolck for her editorial support on this work.

Submitted October 21, 2019; accepted for publication February 20, 2020.

Disclosures: Dr. Keenan has disclosed that she has no financial interests, arrangements, affiliations, or commercial interests with the manufacturers of any products discussed in this article or their competitors. Dr. Tolaney has disclosed that she receives grant/research support from Novartis, Genentech, Eli Lilly, Pfizer, Merck, Exelixis, Eisai, Bristol Meyers Squibb, AstraZeneca, Cyclacel, Immunomedics, Odonate, and Nektar; and that she is a scientific advisor/ consultant for Novartis, Eli Lilly, Pfizer, Merck, AstraZeneca, Eisai, Puma, Genentech, Immunomedics, Nektar, Tesaro, Daiichi-Sankyo, Oncopep, Paxman, Athenex, Abbvie, and Nanostring.

Correspondence: Sara M. Tolaney, MD, MPH, Department of Medical Oncology, Dana-Farber Cancer Institute, 450 Brookline Avenue, Boston, MA 02215. Email: sara_tolaney@dfci.harvard.edu

\section{References}

1. Garrido-Castro AC, Lin NU, Polyak K. Insights into molecular classifications of triple-negative breast cancer: improving patient selection for treatment. Cancer Discov 2019;9:176-198.

2. Topalian SL, Hodi FS, Brahmer JR, et al. Safety, activity, and immune correlates of anti-PD-1 antibody in cancer. N Engl J Med 2012;366:2443-2454.

3. Garon EB, Rizvi NA, Hui R, et al. Pembrolizumab for the treatment of nonsmall-cell lung cancer. N Engl J Med 2015;372:2018-2028.

4. Motzer RJ, Rini BI, McDermott DF, et al. Nivolumab for metastatic renal cell carcinoma: results of a randomized phase II trial. J Clin Oncol 2015;33: 1430-1437.

5. Rosenberg JE, Hoffman-Censits J, Powles T, et al. Atezolizumab in patients with locally advanced and metastatic urothelial carcinoma who have progressed following treatment with platinum-based chemotherapy: a single-arm, multicentre, phase 2 trial. Lancet 2016;387:1909-1920.

6. Wolchok JD, Chiarion-Sileni V, Gonzalez R, et al. Overall survival with combined nivolumab and ipilimumab in advanced melanoma. $\mathrm{N}$ Engl J Med 2017:377:1345-1356.
7. El-Khoueiry AB, Sangro B, Yau T, et al. Nivolumab in patients with advanced hepatocellular carcinoma (CheckMate 040): an open-label, noncomparative, phase $1 / 2$ dose escalation and expansion trial. Lancet 2017 389:2492-2502

8. Kim ST, Cristescu R, Bass AJ, et al. Comprehensive molecular characterization of clinical responses to PD-1 inhibition in metastatic gastric cancer. Nat Med 2018;24:1449-1458.

9. Ribas A, Wolchok JD. Cancer immunotherapy using checkpoint blockade Science 2018;359:1350-1355.

10. Denkert C, von Minckwitz G, Darb-Esfahani S, et al. Tumour-infiltrating lymphocytes and prognosis in different subtypes of breast cancer: a pooled analysis of 3771 patients treated with neoadjuvant therapy. Lancet Oncol 2018;19:40-50.

11. Fehrenbacher L, Spira A, Ballinger M, et al. Atezolizumab versus docetaxel for patients with previously treated non-small-cell lung cancer (POPLAR): a multicentre, open-label, phase 2 randomised controlled trial. Lancet 2016:387:1837-1846. 
12. Loi S, Drubay D, Adams S, et al. Tumor-infiltrating lymphocytes and prognosis: a pooled individual patient analysis of early-stage triplenegative breast cancers. J Clin Oncol 2019;37:559-569.

13. Mittendorf EA, Philips AV, Meric-Bernstam F, et al. PD-L1 expression in triple-negative breast cancer. Cancer Immunol Res 2014;2:361-370.

14. Gatalica Z, Snyder C, Maney T, et al. Programmed cell death 1 (PD-1) and its ligand (PD-L1) in common cancers and their correlation with molecular cancer type. Cancer Epidemiol Biomarkers Prev 2014;23:2965-2970.

15. Luen $S$, Virassamy $B$, Savas $P$, et al. The genomic landscape of breast cancer and its interaction with host immunity. Breast 2016;29:241-250.

16. Schumacher TN, Schreiber RD. Neoantigens in cancer immunotherapy. Science 2015;348:69-74.

17. Yarchoan M, Johnson BA III, Lutz ER, et al. Targeting neoantigens to augment antitumour immunity. Nat Rev Cancer 2017;17:209-222.

18. Nanda R, Chow LQ, Dees EC, et al. Pembrolizumab in patients with advanced triple-negative breast cancer: phase Ib KEYNOTE-012 study. J Clin Oncol 2016;34:2460-2467.

19. Adams S, Schmid P, Rugo HS, et al. Pembrolizumab monotherapy for previously treated metastatic triple-negative breast cancer: cohort A of the phase II KEYNOTE-086 study. Ann Oncol 2019;30:397-404.

20. Adams S, Loi $S$, Toppmeyer $D$, et al. Pembrolizumab monotherapy for previously untreated, PD-L1-positive, metastatic triple-negative breast cancer: cohort B of the phase II KEYNOTE-086 study. Ann Oncol 2019;30: 405-411.

21. Cortes J, Lipatov O, Im S, et al. KEYNOTE-119: phase 3 study of pembrolizumab versus single-agent chemotherapy for metastatic triplenegative breast cancer (mTNBC) [abstract]. Ann Oncol 2019;30(Suppl 5): v851-934.

22. Dirix LY, Takacs I, Jerusalem G, et al. Avelumab, an anti-PD-L1 antibody, in patients with locally advanced or metastatic breast cancer: a phase $1 \mathrm{~b}$ JAVELIN Solid Tumor study. Breast Cancer Res Treat 2018;167:671-686.

23. Emens LA, Cruz C, Eder JP, et al. Long-term clinical outcomes and biomarker analyses of atezolizumab therapy for patients with metastatic triple-negative breast cancer: a phase 1 study. JAMA Oncol 2019;5: 74-82.

24. Ueda S, Saeki T, Takeuchi $H$, et al. In vivo imaging of eribulin-induced reoxygenation in advanced breast cancer patients: a comparison to bevacizumab. Br J Cancer 2016:114:1212-1218.

25. Mariathasan $\mathrm{S}$, Turley SJ, Nickles D, et al. TGF $\beta$ attenuates tumour response to PD-L1 blockade by contributing to exclusion of T cells. Nature 2018;554:544-548.

26. Tolaney SM, Kalinsky K, Kaklamani V, et al. Phase $1 \mathrm{~b} / 2$ study to evaluate eribulin mesylate in combination with pembrolizumab in patients with metastatic triple-negative breast cancer [abstract]. Cancer Res 2018; 78(Suppl):Abstract PD6-13.

27. Adams S, Diamond JR, Hamilton E, et al. Atezolizumab plus nab-paclitaxel in the treatment of metastatic triple-negative breast cancer with 2-year survival follow-up: a phase 1b clinical trial. JAMA Oncol 2019;5:334-342.

28. Schmid P, Adams S, Rugo HS, et al. Atezolizumab and nab-paclitaxel in advanced triple-negative breast cancer. N Engl J Med 2018;379. 2108-2121.

29. Schmid P, Adams S, Rugo HS, et al. IMpassion130: updated overal survival (OS) from a global, randomized, double-blind, placebocontrolled, phase III study of atezolizumab (atezo) + nab-paclitaxel (nP) in previously untreated locally advanced or metastatic triple-negative breast cancer (mTNBC) [abstract]. J Clin Oncol 2019;37(Suppl):Abstract 1003.

30. Kulangara $\mathrm{K}$, Zhang $\mathrm{N}$, Corigliano $\mathrm{E}$, et al. Clinical utility of the combined positive score for programmed death ligand-1 expression and the approval of pembrolizumab for treatment of gastric cancer. Arch Pathol Lab Med 2019;143:330-337.

31. Nanda R, Liu MC, Yau C, et al. Effect of pembrolizumab plus neoadjuvant chemotherapy on pathologic complete response in women with earlystage breast cancer: an analysis of the ongoing phase 2 adaptively randomized I-SPY2 trial [published online February 13, 2020]. JAMA Oncol, doi: 10.1001/jamaoncol.2019.6650

32. Galluzzi L, Buqué A, Kepp O, et al. Immunological effects of conventional chemotherapy and targeted anticancer agents. Cancer Cell 2015;28: 690-714.

33. Zitvogel L, Apetoh L, Ghiringhelli F, et al. Immunological aspects of cancer chemotherapy. Nat Rev Immunol 2008;8:59-73.

34. Loibl S, Untch M, Burchardi N, et al. A randomised phase II study investigating durvalumab in addition to an anthracycline taxane-based neoadjuvant therapy in early triple-negative breast cancer: clinical results and biomarker analysis of GeparNuevo study. Ann Oncol 2019;30:1279-1288.
35. Schmid P, Cortes J, Dent R, et al. KEYNOTE-522: phase 3 study of pembrolizumab (pembro) + chemotherapy (chemo) vs placebo (pbo) + chemo as neoadjuvant treatment, followed by pembro vs pbo as adjuvant treatment of early triple-negative breast cancer (TNBC). Ann Oncol 2019;30(Suppl 5):v851-934

36. Gianni L, Huang CS, Egle D, et al. Pathologic complete response ( $p C R)$ to neoadjuvant treatment with or without atezolizumab in triple negative, early high-risk and locally advanced breast cancer. NeoTRIPaPDL1 Michelangelo randomized study. Presented at the 2019 San Antonio Breast Cancer Symposium; December 10-14, 2019; San Antonio, Texas. Abstract GS3-04.

37. Liu MC, Robinson PA, Yau C, et al. Evaluation of a pembrolizumab-8 cycle neoadjuvant regimen without $A C$ for high-risk early-stage HER2-negative breast cancer: results from the I-SPY 2 TRIAL. Presented at the 2019 San Antonio Breast Cancer Symposium; December 10-14, 2019; San Antonio, Texas. Abstract P3-09-02.

38. Voorwerk L, Slagter M, Horlings HM, et al. Immune induction strategies in metastatic triple-negative breast cancer to enhance the sensitivity to PD-1 blockade: the TONIC trial. Nat Med 2019;25:920-928.

39. LaFleur MW, Muroyama Y, Drake CG, et al. Inhibitors of the PD-1 pathway in tumor therapy. J Immunol 2018;200:375-383.

40. Pantelidou C, Sonzogni O, De Oliveria Taveira M, et al. PARP inhibitor efficacy depends on $\mathrm{CD} 8^{+}$T-cell recruitment via intratumoral STING pathway activation in BRCA-deficient models of triple-negative breast cancer. Cancer Discov 2019;9:722-737.

41. Kraya AA, Maxwell KN, Wubbenhorst B, et al. Genomic signatures predict the immunogenicity of BRCA-deficient breast cancer. Clin Cancer Res 2019;25:4363-4374.

42. Vinayak S, Tolaney SM, Schwartzberg L, et al. Open-label clinical trial of niraparib combined with pembrolizumab for treatment of advanced or metastatic triple-negative breast cancer. JAMA Oncol 2019;5:1132-1140.

43. Senkus-Konefka E, Domchek S, Im SA, et al. Subgroup analysis of olaparib monotherapy versus chemotherapy by hormone receptor and BRCA mutation status in patients with HER2-negative metastatic breast cancer and a germline BRCA mutation: OlympiAD. Eur J Cancer 2018;92(Suppl 3):S19-20.

44. Eiermann W, Rugo HS, Diab S, et al. Analysis of germline BRCA1/2 mutated (gBRCAmut) hormone receptor-positive $(\mathrm{HR}+)$ and triple negative breast cancer (TNBC) treated with talazoparib (TALA) [abstract]. J Clin Oncol 2018;36(Suppl):Abstract 1070.

45. Domchek S, Postel-Vinay S, Im S, et al. Phase II study of olaparib $(\mathrm{O})$ and durvalumab (D) (MEDIOLA): updated results in patients (pts) with germline BRCA-mutated (gBRCAm) metastatic breast cancer (MBC). Ann Oncol 2019;30(Suppl 5):v475-532.

46. Peng W, Chen JQ, Liu C, et al. Loss of PTEN promotes resistance to T cellmediated immunotherapy. Cancer Discov 2016;6:202-216.

47. George $S$, Miao D, Demetri GD, et al. Loss of PTEN is associated with resistance to anti-PD-1 checkpoint blockade therapy in metastatic uterine leiomyosarcoma. Immunity 2017;46:197-204.

48. Crompton JG, Sukumar M, Roychoudhuri R, et al. Akt inhibition enhances expansion of potent tumor-specific lymphocytes with memory cell characteristics. Cancer Res 2015;75:296-305.

49. Schmid P, Loirat $D$, Savas $P$, et al. Phase $1 \mathrm{~b}$ study evaluating a triplet combination of ipatasertib, atezolizumab, and paclitaxel or nab-paclitaxel as first-line therapy for locally advanced/metastatic triple-negative breast cancer [abstract]. Cancer Res 2019;79(13 Suppl):Abstract CT049.

50. Loi S, Dushyanthen S, Beavis PA, et al. RAS/MAPK activation is associated with reduced tumor-infiltrating lymphocytes in triple-negative breast cancer: therapeutic cooperation between MEK and PD-1/PD-L1 immune checkpoint inhibitors. Clin Cancer Res 2016;22:1499-1509.

51. Brufsky A, Kim SB, Zvirbule Z, et al. Phase II COLET study: atezolizumab (A) + cobimetinib (C) + paclitaxel (P)/nab-paclitaxel (nP) as first-line (1L) treatment (tx) for patients (pts) with locally advanced or metastatic triplenegative breast cancer (mTNBC) [abstract]. J Clin Oncol 2019;37(Suppl): Abstract 1013

52. Charych DH, Hoch U, Langowski JL, et al. NKTR-214, an engineered cytokine with biased IL2 receptor binding, increased tumor exposure, and marked efficacy in mouse tumor models. Clin Cancer Res 2016;22: 680-690.

53. Diab A, Hurwitz ME, Cho DC, et al. NKTR-214 (CD-122-biased agonist) plus nivolumab in patients with advanced solid tumors: preliminary phase 1/2 results of PIVOT [abstract]. J Clin Oncol 2018;36(Suppl):Abstract 3006.

54. Bentebibel $S E$, Hurwitz ME, Bernatchez $C$, et al. A first-in-human study and biomarker analysis of NKTR-214, a novel IL2R $\beta \gamma$-biased cytokine, in patients with advanced or metastatic solid tumors. Cancer Discov 2019;9: 711-721. 
55. Ribas A, Medina T, Kummar S, et al. SD-101 in combination with pembrolizumab in advanced melanoma: results of a phase $\mathrm{lb}$, multicenter study. Cancer Discov 2018;8:1250-1257.

56. Isakoff SJ, Tolaney SM, Tung NM, et al. A phase $1 \mathrm{~b}$ study of safety and immune response to PVX-410 vaccine alone and in combination with durvalumab (MEDI4736) in HLA-A2+ patients following adjuvant therapy for stage 2/3 triple negative breast cancer [abstract]. J Clin Oncol 2017; 35(Suppl):Abstract TPS1126.

57. Kalli KR, Block MS, Kasi PM, et al. Folate receptor alpha peptide vaccine generates immunity in breast and ovarian cancer patients. Clin Cancer Res 2018;24:3014-3025.

58. Liu XS, Mardis ER. Applications of immunogenomics to cancer. Cell 2017; 168:600-612.

59. Maeng $\mathrm{H}$, Terabe M, Berzofsky JA. Cancer vaccines: translation from mice to human clinical trials. Curr Opin Immunol 2018;51:111-122.

60. Hernandez-Aya LF, Gao F, Goedegebuure PS, et al. A randomized phase II study of nab-paclitaxel + durvalumab + neoantigen vaccine versus nabpaclitaxel + durvalumab in metastatic triple-negative breast cancer (mTNBC) [abstract]. J Clin Oncol 2019;37(Suppl):Abstract TPS1114.

61. Zacharakis $\mathrm{N}$, Chinnasamy $\mathrm{H}$, Black $\mathrm{M}$, et al. Immune recognition of somatic mutations leading to complete durable regression in metastatic breast cancer. Nat Med 2018;24:724-730.

62. Tchou J, Zhao Y, Levine BL, et al. Safety and efficacy of intratumoral injections of chimeric antigen receptor (CAR) T cells in metastatic breast cancer. Cancer Immunol Res 2017;5:1152-1161.

63. Morello A, Sadelain M, Adusumilli PS. Mesothelin-targeted CARs: driving T cells to solid tumors. Cancer Discov 2016;6:133-146.

64. Le DT, Durham JN, Smith KN, et al. Mismatch repair deficiency predicts response of solid tumors to PD-1 blockade. Science 2017;357:409-413.

65. Reck M, Rodríguez-Abreu D, Robinson AG, et al. Pembrolizumab versus chemotherapy for PD-L1-positive non-small-cell lung cancer. N Engl J Med 2016;375:1823-1833.

66. Rugo HS, Loi S, Adams S, et al. Performance of PD-L1 immunohistochemistry (IHC) assays in unresectable locally advanced or metastatic triple-negative breast cancer (mTNBC): post-hoc analysis of IMpassion130 [abstract]. Ann Oncol 2019;30(Suppl):v858-859.

67. Rimm DL, Han G, Taube JM, et al. A prospective, multi-institutional, pathologist-based assessment of 4 immunohistochemistry assays for PDL1 expression in non-small cell lung cancer. JAMA Oncol 2017;3: 1051-1058.

68. Ribas A, Hu-Lieskovan S. What does PD-L1 positive or negative mean? J Exp Med 2016;213:2835-2840.

69. Schmid P, Park YH, Ferreira M, et al. Keynote-522 study of pembrolizumab + chemotherapy vs placebo + chemotherapy as neoadjuvant treatment, followed by pembrolizumab vs placebo as adjuvant treatment of early triple-negative breast cancer: pathologic complete response in key subgroups. Presented at the 2019 San Antonio Breast Cancer Symposium; December 10-14, 2019; San Antonio, Texas. Abstract GS3-03.

70. Rizvi NA, Hellmann MD, Snyder A, et al. Cancer immunology. Mutational landscape determines sensitivity to PD-1 blockade in non-small cell lung cancer. Science 2015;348:124-128.
71. Carbone DP, Reck M, Paz-Ares $\mathrm{L}$, et al. First-line nivolumab in stage IV or recurrent non-small-cell lung cancer. N Engl J Med 2017;376:2415-2426.

72. Hellmann MD, Callahan MK, Awad MM, et al. Tumor mutational burden and efficacy of nivolumab monotherapy and in combination with ipilimumab in small-cell lung cancer. Cancer Cell 2018;33:853-861.e854.

73. Cristescu R, Mogg R, Ayers M, et al. Pan-tumor genomic biomarkers for PD-1 checkpoint blockade-based immunotherapy. Science 2018;362:eaar3593.

74. Ott PA, Bang YJ, Piha-Paul SA, et al. T-cell-inflamed gene-expression profile, programmed death ligand 1 expression, and tumor mutational burden predict efficacy in patients treated with pembrolizumab across 20 cancers: KEYNOTE-028. J Clin Oncol 2019;37:318-327.

75. Samstein RM, Lee $\mathrm{CH}$, Shoushtari AN, et al. Tumor mutational load predicts survival after immunotherapy across multiple cancer types. Nat Genet 2019;51:202-206.

76. Barroso-Sousa R, Jain E, Cohen O, et al. Prevalence and mutational determinants of high tumor mutation burden in breast cancer. Ann Oncol 2020;31:387-394.

77. Barroso-Sousa R, Jain E, Kim D, et al. Determinants of high tumor mutational burden (TMB) and mutational signatures in breast cancer [abstract]. J Clin Oncol 2018;36(Suppl):Abstract 1010.

78. Chen R, Zinzani PL, Fanale MA, et al. Phase II study of the efficacy and safety of pembrolizumab for relapsed/refractory classic Hodgkin lymphoma. J Clin Oncol 2017;35:2125-2132.

79. Barroso-Sousa R, Keenan TE, Pernas $S$, et al. Tumor mutational burden and PTEN alterations as molecular correlates of response to PD-1/L1 blockade in metastatic triple-negative breast cancer [published online February 4, 2020]. Clin Cancer Res, doi: 10.1158/1078-0432.CCR-19-3507

80. Schmid P, Cruz C, Braiteh F, et al. Abstract 2986: atezolizumab in metastatic TNBC (mTNBC): long-term clinical outcomes and biomarker analyses [abstract]. Cancer Res 2017;77(13 Suppl):Abstract 2986.

81. Loi S, Winer E, Lipatov O, et al. Abstract PD5-03: relationship between tumor-infiltrating lymphocytes (TILs) and outcomes in KEYNOTE-119 study of pembrolizumab vs chemotherapy for previously treated metastatic triple-negative breast cancer (mTNBC) [abstract]. Cancer Res 2020 80(4 Suppl):Abstract PD5-03.

82. Emens LA, Loi S, Rugo HS, et al. Abstract GS1-04: IMpassion130: efficacy in immune biomarker subgroups from the global, randomized, double-blind, placebo-controlled, phase III study of atezolizumab + nab-paclitaxel in patients with treatment-naïve, locally advanced or metastatic triple-negative breast cancer [abstract]. Cancer Res 2019; 79(4 Suppl):Abstract GS1-04.

83. Blenman K, Li X, Marczyk M, et al. Predictive markers of response to durvalumab concurrent with nab-paclitaxel and dose dense doxorubicin cyclophosphamide (ddAC) neoadjuvant therapy for triple negative breast cancer (TNBC). Presented at the 42nd Annual SABCS; December 10-14, 2019; San Antonio, Texas. Abstract 1870.

84. Sade-Feldman M, Jiao YJ, Chen JH, et al. Resistance to checkpoint blockade therapy through inactivation of antigen presentation. Nat Commun 2017;8:1136. 